\title{
Research Paper: Methanol Poisoning and Its Treatment
}

\author{
Fares Najari $^{* *}$, Ideh Baradaran ${ }^{2}$, Dorsa Najari ${ }^{3}$ (D)
}

1. Forensic Medicine, Clinical Toxicology, Shahid Beheshti University of Medical Sciences, Tehran, Iran.

2. Forensic Medicine, Karaj Forensic Medicine Center, Karaj, Alborz, Iran.

3. School of Medicine, Shahid Beheshti University of Medical Sciences, Tehran, Iran.

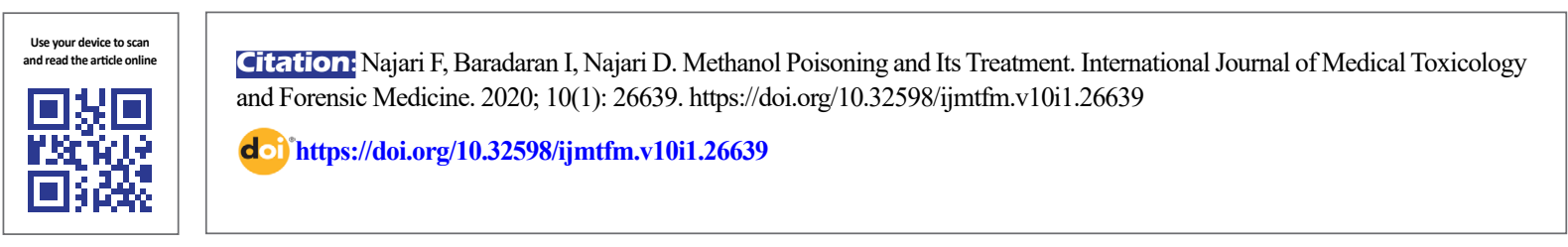

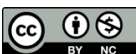

Article info:

Received: 08 Apr 2019

First Revision: 25 Apr 2019

Accepted: 06 Sep 2019

Published: 03 Apr 2020

\section{Keywords:}

Methanol, Alcohol, Poisoning, Toxicity

\section{ABSTRACT}

Background: Methanol is a toxic alcohol found in illegal liquor, and its poisoning may cause death if not treated timely and properly. In 2018, methanol toxicity in Iran increased dramatically. Regarding the high prevalence of methanol toxicity, its high mortality rate, and the cost of treatment as well as the importance of timely diagnosis in the treatment of this condition, we decided to review methanol toxicity, its signs, symptoms, diagnosis, and treatment.

Evidence Acquisition: We searched for articles on reliable databases such as Embase and Medline from January 2018 to May 2018 using the following keywords: "methanol," "toxic alcohol," and "ethanol toxicity treatment."

Results: This review discusses how to diagnose methanol toxicity by using clinical signs and symptoms and laboratory examinations; it also suggests new treatments. Meanwhile, we provide alternatives for diagnosis in case of shortages of tests in the emergency department.

Conclusion: Diagnosis of ethanol toxicity due to non-specific signs and symptoms, late patient's referral, and lack of proper history-taking can be very difficult and delay the onset of treatment. There is also no clear scientific evidence as to whether ethanol or fomepizole should be used as the first choice of therapy for methanol toxicity because there is no direct comparison between these two antidotes regarding their effectiveness and safety. The appropriate antidote is chosen depending on the availability, cost, accessibility of hemodialysis, and the physician's experience. If the therapist does not have enough experience, it is easier to use fomepizole.

\section{Context}

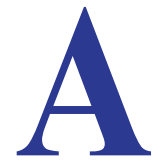

lcohol consumption is an undeniable problem, and unfortunately, alcohol consumption is the underlying cause of many social harms [1]. Methanol is a kind of toxic alcohol that is present in glass cleaners, paints, varnishes, photocopy machine inks, bottles of antifreeze, and perfumes, and usually in illegal liquor [2, 3]. Therefore, after consuming alcoholic liquors containing methanol, people are accidentally exposed to methanol toxicity, which is a deadly condition.

\footnotetext{
* Corresponding Author:

Fares Najari, MD.

Address: Forensic Medicine, Clinical Toxicology, Shahid Beheshti University of Medical Sciences, Tehran, Iran.

Tel: +98 (912) 3195140

E-mail: najari.hospital@sbmu.ac.ir
} 
Methanol toxicity, if not treated timely and properly, causes death [4]. Considering the reported cases of mass epidemics of methanol toxicity in some Iranian cities [5], it is essential to understand the principles of diagnosis and treatment of methanol toxicity. Methanol toxicity is one of the emergency conditions that its timely diagnosis can reduce mortality, blindness, and other physical and mental problems.

In 2018, methanol toxicity in Iran increased dramatically. According to the Iranian Legal Medicine Organization, 96 people died of alcohol consumption from September 1 to October 15, 2018 [5]. From September 7 to October 7, 2018, we witnessed an increase in the incidence of severe alcohol toxicity. Nearly in four weeks, 768 people were poisoned with illegal and nonstandard alcohol. Of them, 96 people died of alcohol poisoning, and 76 of methanol toxicity. The methanol mortality rate was $10.1 \%$. Eighty-six cases of mortality were male, and 10 were female. The forensic organization of Iran and the police collected samples of counterfeit beverages, and the results of the experiments showed that they contained a high concentration of methanol, which is the leading cause of high toxicity [6]. Given the high incidence, mortality rate, and cost of treatment of methanol toxicity, we sought to perform a review study on this topic.

Type of study:

This research is a review study discussing the symptoms of methanol toxicity, its diagnosis, and treatment.

\section{Evidence Acquisition}

We searched for articles on reliable databases such as Embase and Medline from January 2018 to May 2019 using the following keywords: "methanol," "toxic alcohol," and "ethanol toxicity treatment."

\section{Discussion}

Absorption, distribution, and metabolism of methanol

Methanol, like other alcohols, is absorbed from the gastrointestinal tract immediately after ingestion. Methanol is not toxic by itself, but its metabolites produced by the alcohol dehydrogenase (ADH) enzyme are considered toxic. Its absorption half-life is 5 minutes, and it is well dissolved in the body's water and reaches its maximum serum concentration within 30-60 minutes. Methanol is metabolized by the $\mathrm{ADH}$ enzyme and converted to formaldehyde. The formaldehyde is also converted to formic acid by aldehyde dehydrogenase (ALDH) (Figure 1) [7]. The produced formic acid inhibits cytochrome oxidase in the electron transport chain, which ultimately leads to cellular dysfunction and organ failure. The accumulation of toxic metabolites affects the end organs, including the retina and the brain, leading to blindness and lesion in the basal ganglia. Also, these metabolites increase the anion gap. Therefore, patients with methanol toxicity present to the hospital with metabolic acidosis and high anion gap [8].
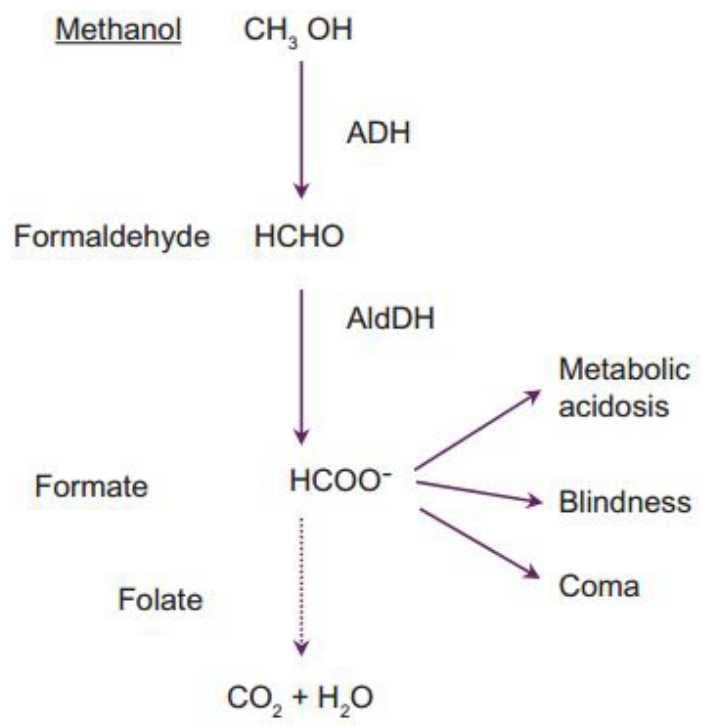

Figure 1. Methanol metabolism 


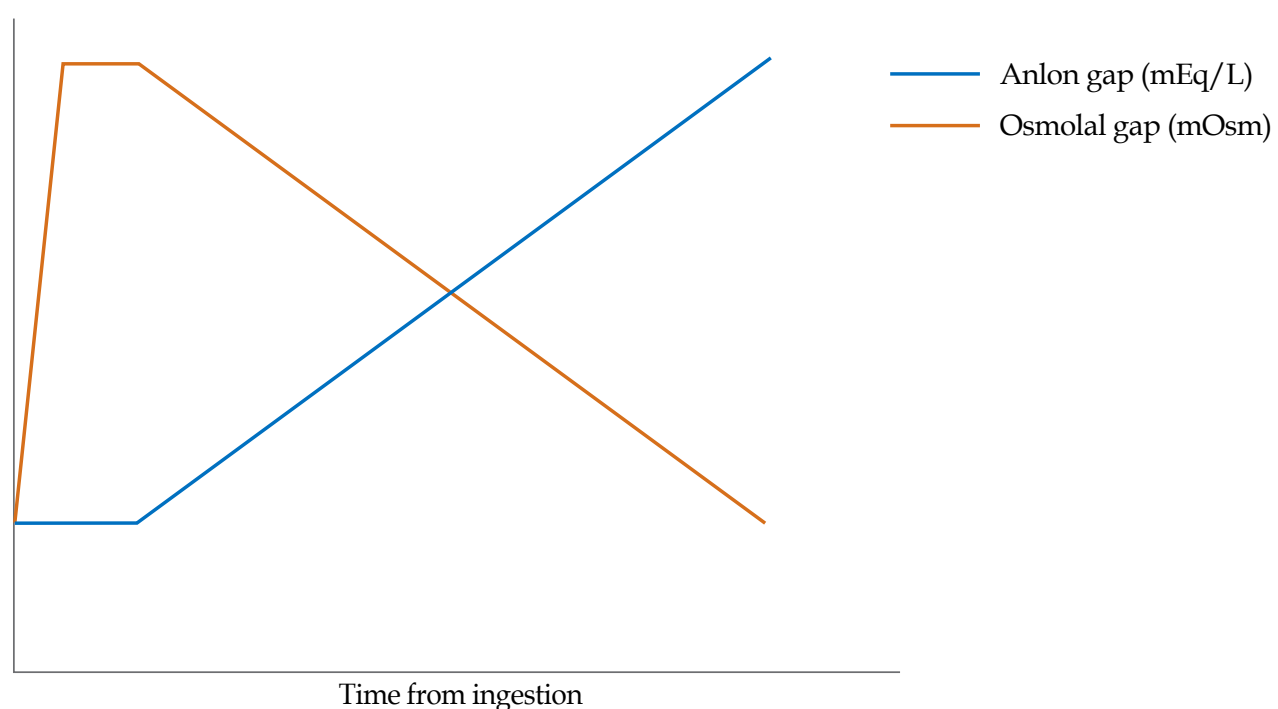

Figure 2. Anion and Osmolal Gaps Variation after ingestion

Patients can present with various non-specific symptoms $12-24 \mathrm{~h}$ after methanol ingestion. Common symptoms include abdominal pain, headache, nausea, vomiting, and visual disturbances, which may be described as cloudy or blurry vision [9].

The presence of severe metabolic acidosis with an anionic and osmolality gap is strongly suggestive of the existence of methanol or ethylene glycol toxicity, and the clinical manifestations and mortality of patients are significantly associated with metabolic acidosis and central nervous system weakening [9]. In the neurological examination, the poisoned may have impaired walking or imbalance, and cerebral hemorrhage is a rare condition that has been reported in some cases [10]. Timely treatment of methanol toxicity is vital to prevent mortality [11].

\section{Diagnosis}

Measurement of serum levels of methanol in Iran is not available everywhere; moreover, the negative serum level does not entirely reject methanol toxicity because the level of methanol depends to some extent on the time of methanol consumption. For example, if a patient who has consumed methanol refers to a clinic several hours after methanol metabolism, a negative serum level is reported. Detection of methanol toxicity is a combination of clinical symptoms and laboratory findings. The presence of acidemia in a patient can be a sign of poisoning with toxic alcohol. Serial serum bicarbonate change is more helpful than bicarbonate itself. A review study published by Patrick chow Yuen Ng and his colleagues in 2018 suggests that a person suspected of methanol intoxication should be examined every 2-4 hours for 12 hours [9].

Where serum levels are unavailable, reduced bicarbonate without justifiable causes may suggest an accumulation of organic acid metabolites from toxic alcohol metabolism. The osmolality gap decreases as a result of methanol metabolism, and anion gap increases as the organic acid metabolites accumulate [7]. The progressive reduction of the osmolality gap with the continued rise of the anion gap in an intoxicated patient may suggest toxic alcohol consumption. Anion gap and osmolality gap depend on time [7]. Early after eating methanol, the osmolality gap is high due to the presence of methanol in the blood.

When methanol is metabolized to organic acid, the anion gap starts to increase, and the osmolality gap begins to decrease [7]. This dynamic process of toxic alcohol metabolism is essential at the clinical level, as the occurrence or non-occurrence of anion gap or osmolality gap alone cannot confirm the diagnosis of toxic alcohol poisoning, and these should be evaluated clinically in each patient. Severe acidemia with no known reason can be observed in those with methanol toxicity who have referred late, and the osmolality gap and anion gap have sufficient time to get normalized. This article considers bicarbonate less than 12 as severe acidosis [7] (Figure 2).

\section{Treatment}

Gastric lavage, or the use of activated charcoal due to the rapid absorption of alcohol, does not affect alcohol 
poisoning. The basis of methanol intoxication comprises the use of an antidote (fomepizole or ethanol) to prevent the oxidation of methanol, folic acid to facilitate formic acid catabolism, acidosis modification, and hemodialysis to accelerate the removal of methanol [12]. Sodium bicarbonate is suggested to correct acute acidosis $(\mathrm{pH}<7.3)[7]$.

\section{Indications for starting antidotes}

The evidence for starting antidotes are the existence of severe metabolic acidosis (base deficit $<10 \mathrm{mmol} / \mathrm{L}$ ) or the presence of symptoms of organ dysfunction. In the absence of these two criteria, the following criteria are used (Table1) [13]:

Two types of antidotes are recommended for inhibiting methanol metabolism mediated by the ADH enzyme. The first one is ethanol, which is a competitive $\mathrm{ADH}$ substrate. The ADH's affinity for ethanol is 10 times higher than its affinity for methanol, which also makes ethanol useful as a competitive inhibiting agent, leading to a reduction in the production of toxic methanol metabolites [14]. Second is fomepizole, which is an ADH inhibitor. Hemodialysis is considered as an integral part of the treatment of severe methanol toxicity. It aims to remove both the primary combination and its toxic metabolites and to correct acidosis of metabolites and electrolyte disorders, and thus reduce the duration of treatment with antidotes and hospitalization [7].

\section{Hemodialysis criteria}

The criteria for hemodialysis are primary methanol plasma concentration greater than $500 \mathrm{mg} / \mathrm{L}$, severe metabolic acidosis $(\mathrm{pH}<7.3)$, kidney failure, treatmentresistant electrolyte disorders, visual impairment, and abnormal vital signs despite supportive care. An appropriate therapeutic threshold is serum bicarbonate concentrations of less than $12 \mathrm{mEq} / \mathrm{L}$, although a few articles are supporting this issue $[12,15]$.

\section{Ethanol or fomepizole}

Fomepizole is more likely to inhibit ADH, and its duration of action is longer. It is also easier to use. It has an initial dose that lasts every 12 hours with bolus doses and does not need to check the serum level. Fomepizole is administered at a dose of $15 \mathrm{mg} / \mathrm{kg}$ intravenously within 30 minutes and continues with $10 \mathrm{mg} / \mathrm{kg}$ every 12 hours until the methanol level reaches below $30 \mathrm{mg} /$ $\mathrm{dL}$. The dose of fomepizole should be increased after 48 hours to overcome its clearance by the p450 enzymes. Fomepizole is also removed by hemodialysis, so its dosage should be increased during hemodialysis.

There are two protocols to compensate for the reduction of fomepizole due to its withdrawal. The American protocol suggests that the injection intervals be reduced from 12 hours to 4 hours, while the European protocol suggests the continuation of the intravenous infusion at a dose of $1-1.5 \mathrm{mg} / \mathrm{kg} / \mathrm{h}$ after the loading dose throughout the hemodialysis period $[3,16,17]$. Fomepizole is well-tolerated, although its side effects such as injection site irritation, nausea, dizziness, tachycardia, headache, eosinophilia, agitation, and seizure have been reported $[13,18]$.

Ethanol is more likely to bind to ADHs than toxic alcohol. The presence of ethanol prevents the formation of toxic metabolites, and if fomepizole is not available, it may be enough for the ADH blocking [4]. During methanol intoxication treatment, monitoring blood levels of ethanol is required every 1-2 hours. To achieve the therapeutic goals, the level of ethanol in the blood should be kept within the range of $100 \mathrm{mg} / \mathrm{dL}(22 \mathrm{mmol} / \mathrm{L})$ [13].

To achieve this goal, the loading dose is initially given at a rate of $0.6 \mathrm{~g} / \mathrm{kg}(13 \mathrm{mmol} / \mathrm{kg})$. Then, the maintenance dose is given to keep the level of blood ethanol within the target range (this maintenance dose is 66$154 \mathrm{mg} / \mathrm{kg}$ or $1.4-3.3 \mathrm{mmol} / \mathrm{kg}$ ), given orally or intravenously. Chronic consumption of alcohol accelerates the metabolism of ethanol; thus, the ethanol mainte-

Table 1. Indications for starting antidotes

\section{Criteria}

1. Blood methanol level $>10 \mathrm{mmol} / \mathrm{L}(32 \mathrm{mg} / \mathrm{dL})$

2. Conclusive or even suspicious history of recent alcohol use with osmolality gap $>25 \mathrm{mOsmol} / \mathrm{kg}$

3. Definitive or suspicious history of alcohol consumption with two or more of the following criteria:

Arterial $\mathrm{pH}<7.3$, serum bicarbonate $<20 \mathrm{mmol} / \mathrm{L}$, os- molality gap $>25 \mathrm{mOsmol} / \mathrm{kg}$, and visual impairment 
Table 2. Indications for methanol toxicity treatment with ethanol or fomepizole

\section{Criteria}

1. Documented serum methanol concentration $>20 \mathrm{mg} / \mathrm{dL}$

2. Documented history of ingestion of methanol and os- molality gap over $10 \mathrm{mosm} / \mathrm{kg} \mathrm{H} 2 \mathrm{O}$

3. History or strong clinical suspicion * of methanol tox- icity that the patient has referred to the clinic within 72 hours after ingestion with two of the following:

A. Arterial $\mathrm{pH}<7.3$

B. Serum bicarbonate $<20 \mathrm{mEq} / \mathrm{L}$

C. Osmolality gap more than $10 \mathrm{mosm} / \mathrm{kg} \mathrm{H} \mathrm{O}$

International Journal of
Medical Toxicology \& forensic Medicin * In cases of the epidemic condition, we recommend that treatment be started with any level of clinical suspicion of toxicity.

nance dose should be increased in chronic consumers. Ethanol is also withdrawn by hemodialysis, as is the case with fomepizole, so dosage should be increased during hemodialysis. Maintenance dosing is doubled during dialysis [3].

\section{Complications of ethanol therapy}

Hypoglycemia, changes in consciousness level, liver toxicity, or pancreatitis may occur as the complications of ethanol therapy. Therefore, in addition to the level of ethanol in the blood, the level of consciousness of the patient and blood glucose should also be checked regularly [16].

The benefits of ethanol therapy lie in its cost-effectiveness and availability. For auxiliary treatment, folic acid is used, which converts formic acid into water and carbon dioxide by affecting the tetrahydrofolate synthase enzyme. The function of this enzyme is dependent on folic acid [15].

Table 1 presents the indications for treatment with ethanol and fomepizole in methanol toxicity and the algorithm for treating this condition. Besides having dialysis conditions, if a patient needs to receive ethanol, it is necessary to at least double the dose of ethanol during hemodialysis with respect to the removal of ethanol through dialysis (Table 2).

\section{Conclusion}

Diagnosis of ethanol intoxication due to its non-specific signs and symptoms, delayed patient's referral, and lack of proper patient history can be challenging and postpone the treatment. There is also no definitive scientific evidence as to whether ethanol or fomepizole should be used as the first choice of therapy for methanol toxicity because there is no direct comparison between these two antidotes regarding their effectiveness and safety. The proper antidote is selected based on availability, cost, accessibility of hemodialysis, and physician's experience. If the therapist does not have enough experience, it is easier to use fomepizole.

\section{Ethical Considerations}

\section{Compliance with ethical guidelines}

All ethical principles were considered in this article. The participants were informed about the purpose of the research and its implementation stages; they were also assured about the confidentiality of their information. Moreover, they were allowed to leave the study whenever they wish, and if desired, the results of the research would be available to them.

\section{Funding}

This research did not receive any specific grant from funding agencies in the public, commercial, or not-forprofit sectors.

\section{Author's contributions}

All authors contributed to designing, performing, and writing all parts of the article.

\section{Conflict of interest}

The authors declared no conflict of interest.

\section{Acknowledgements}

We wish to thank the Head of Department of Forensic Medicine, School of Medicine, Shahid Beheshti Univer- 
sity of Medical Sciences and the forensic specialists who contributed to carrying out article

\section{References}

[1] Haghdoost AA, Emami M, Esmaili M, Soberinia A, Nezhad Ghaderi M, Mehrolhassani MH. Survey the status and causes of alcohol consumption: A case study of the epidemic alcohol poisoning in Rafsanjan in 2013. Journal of Rafsanjan University of Medical Sciences. 2015; 13(10):991-1006.

[2] Moral AR, Çankayalı İ, Sergin D, Boyacılar Ö. Neuromuscular functions on experimental acute methanol intoxication. Turkish Journal of Anaesthesiology and Reanimation. 2015 43(5):337-43. [DOI:10.5152/TJAR.2015.13471] [PMID] [PMCID]

[3] Ashurst JV, Nappe TM. Methanol toxicity. StatPearls. Treasure Island (FL): StatPearls Publishing; 2019-2020.

[4] Rietjens SJ, De Lange DW, Meulenbelt J. Ethylene glycol or methanol intoxication: which antidote should be used, fomepizole or ethanol. The Netherlands Journal of Medicine. 2014 72(2):73-9.

[5] Iranian Legal Medicine Organization [Internet]. 2020 [Updated 2010 Mar 15]. Available from: www.LMO.ir.

[6] Aghababaeian H, Araghi Ahvazi L, Ostadtaghizadeh A. The methanol poisoning outbreaks in Iran 2018. Alcohol and Alcoholism. 2019; 54(2):128-30. [DOI:10.1093/alcalc/agz005] [PMID]

[7] Mégarbane B. Treatment of patients with ethylene glycol or methanol poisoning: focus on fomepizole. Open Access Emergency Medicine (OAEM). 2010; 2:67-75. [DOI:10.2147/ OAEM.S5346] [PMID] [PMCID]

[8] Liesivuori J, Savolainen H. Methanol and formic acid toxicity: Biochemical Mechanisms. Pharmacol Toxicol. 1991; 69:157-63. [DOI:10.1111/j.1600-0773.1991.tb01290.x] [PMID]

[9] Ng PC, Long BJ, Davis WT, Sessions DJ, Koyfman A. Toxic alcohol diagnosis and management: An emergency medicine review. Internal and Emergency Medicine. 2018; 13(3):375-83. [DOI:10.1007/s11739-018-1799-9] [PMID]

[10] Hassanian-Moghaddam H, Bahrami-Motlagh H, Zamani $\mathrm{N}$, Fazeli SA, Behnam B. Intracranial hemorrhage in methanol toxicity: Challenging the probable heparin effect during hemodialysis. Journal of Research in Pharmacy Practice. 2017 6(3):186-9. [DOI:10.4103/jrpp.JRPP_17_39] [PMID] [PMCID]

[11] Beauchamp GA, Valento M, Kim J. Toxic alcohol ingestion: Prompt recognition and management in the emergency department [digest]. Emergency Medicine Practice. 2016; 18(Suppl 9):S1-S2.

[12] Barceloux DG, Bond GR, Krenzelok EP, Cooper H, Vale JA. American academy of clinical toxicology practice guidelines on the treatment of methanol poisoning. Journal of Toxicology: Clinical Toxicology. 2002; 40(4):415-46. [DOI:10.1081/ CLT-120006745] [PMID]
[13] McMartin K, Jacobsen D, Hovda KE. Antidotes for poisoning by alcohols that form toxic metabolites. British Journal of Clinical Pharmacology. 2016; 81(3):505-15. [DOI:10.1111/ bcp.12824] [PMID] [PMCID]

[14] Gallagher N, Edwards FJ. The diagnosis and management of toxic alcohol poisoning in the emergency department: A review article. Advanced Journal of Emergency Medicine. 2019; 3(3):e28. [DOI:10.22114/ajem.v0i0.153]. [PMID] [PMCID]

[15] Faessel H, Scherrmann JM, Houze P, Baud FJ. 4-methylpyrazole monitoring during haemodialysis of ethylene glycol intoxicated patients. European Journal of Clinical Pharmacology. 1995; 49(3):211-3. [DOI:10.1007/BF00192381] [PMID]

[16] Jobard E, Harry P, Turcant A, Marie Roy P, Allain P. 4-methylpyrazole and hemodialysis in ethylene glycol poisoning. Journal of Toxicology: Clinical Toxicology. 1996; 34(4):373-7. [DOI:10.3109/15563659609013806] [PMID]

[17] Brent J. Fomepizole for ethylene glycol and methanol poisoning. The New England Journal of Medicined. 2009; 360(21):2216-23. [DOI:10.1056/NEJMct0806112] [PMID]

[18] Jacobsen D, McMartin KE. Antidotes for methanol and ethylene glycol poisoning. Clinical Toxicology. 1997; 35(2):12743. [DOI:10.3109/15563659709001182] [PMID] 